\title{
Tratamento de efluentes provenientes da fabricação de lentes solares
}

\author{
Wastewater treatment from the manufacture of solar lenses \\ Tratamiento de efluentes de la fabricación de lente de sol
}

Recebido: 13/08/2021 | Revisado: 18/08/2021 | Aceito: 23/08/2021 | Publicado: 24/08/2021

Juliane C. Forti
ORCID: https://orcid.org/0000-0001-8084-2336
Universidade Estadual Paulista, Brasil
E-mail: juliane.forti @unesp.br
Yasmin S. Tadayozzi
ORCID: https://orcid.org/0000-0001-8938-014X
Universidade Estadual Paulista, Brasil
E-mail: yasmin.tadayozzi @unesp.br
Tamara Cardeal C. da Silva
ORCID: https://orcid.org/0000-0001-8371-7979
Universidade Estadual Paulista, Brasil
E-mail: tamara.cardeal@ gmail.com
Carolina R. V. de Andrade
ORCID: https://orcid.org/0000-0002-5344-2991
Universidade Estadual Paulista, Brasil
E-mail: carolinarva01 @outlook.com
Felipe A. dos Santos
ORCID: https://orcid.org/0000-0001-7264-3396
Universidade Estadual Paulista, Brasil
E-mail: felipe.andre@ @unesp.br

\section{Resumo}

Este trabalho é uma parceria com uma empresa de produtos oftálmicos que gera efluentes contendo uma mistura de três corantes dispersos: azul 56, amarelo 54 e vermelho 60, todos da classe das antraquinonas. Os corantes dessa classe são resistentes a degradação devido a sua estrutura aromática, a qual retêm a cor por longos períodos de tempo. $\mathrm{O}$ tratamento destes corantes é de extrema importância devido à sua toxicidade, podendo apresentar comportamento cancerígeno e mutagênico. $\mathrm{O}$ objetivo deste trabalho foi oxidar os compostos orgânicos poluentes usando processos oxidativos avançados. Para isso, foram realizadas eletrólises a corrente constante usando eletrodos de óxidos como catalisadores heterogêneos juntamente com o reagente de Fenton $\left(\mathrm{Fe}^{2+}+\mathrm{H}_{2} \mathrm{O}_{2}\right)$. A descoloração e oxidação foram monitoradas por espectrometria UV-Visível, cromatografia líquida de alta eficiência e carbono orgânico total. O tratamento aplicado permitiu uma rápida degradação dos grupos cromóforos e houve grande influência da concentração do peróxido de hidrogênio na velocidade dessa degradação. Os resultados foram satisfatórios, uma vez que a coloração foi eliminada e os produtos da oxidação identificados não apresentam toxicidade e podem facilmente tratados por métodos tradicionais.

Palavras-chave: Corantes; Antraquinona; Eletro-Fenton; Efluente industrial; Processo oxidativo avançado.

\begin{abstract}
This work is a partnership with an ophthalmic products company that generates effluents containing a mixture of three dyes: blue 56, yellow 54 and red 60, all in the anthraquinone class. Dyes in this class are resistant to degradation due to their aromatic structure, which retains color for long periods of time. The treatment of these dyes is extremely important due to their toxicity, and may present carcinogenic and mutagenic behavior. The objective of this work was to oxidize polluting organic compounds using advanced oxidative processes. For this, constant current electrolysis was performed using oxide electrodes as heterogeneous catalysts together with Fenton's reagent $\left(\mathrm{Fe}^{2+}+\mathrm{H}_{2} \mathrm{O}_{2}\right)$. Discoloration and oxidation were monitored by UV-Visible spectrometry, high performance liquid chromatography and total organic carbon. The applied treatment allowed a rapid degradation of the chromophore groups and there was a great influence of the hydrogen peroxide concentration on the speed of this degradation. The results were satisfactory, since the coloration was eliminated and the oxidation products identified do not present toxicity and can be easily treated by traditional methods.
\end{abstract}

Keywords: Dyes; Anthraquinone; Electro-Fenton; Industrial effluent; Advanced oxidative process.

\section{Resumen}

Este trabajo es una asociación con una empresa de productos oftálmicos que genera efluentes que contienen una mezcla de tres tintes: azul 56, amarillo 54 y rojo 60, todos en la clase de antraquinonas. Los tintes de esta clase son resistentes a la degradación debido a su estructura aromática, que retiene el color durante largos períodos de tiempo. 
El tratamiento de estos colorantes es de suma importancia por su toxicidad, pudiendo presentar un comportamiento cancerígeno y mutagénico. El objetivo de este trabajo fue oxidar compuestos orgánicos contaminantes mediante procesos oxidativos avanzados. Para ello, se realizó una electrólisis en corriente constante utilizando electrodos de óxido como catalizadores heterogéneos junto con el reactivo de Fenton $\left(\mathrm{Fe}^{2+}+\mathrm{H}_{2} \mathrm{O}_{2}\right)$. La decoloración y oxidación se controlaron mediante espectrometría UV-visible, cromatografía líquida de alta resolución y carbono orgánico total. El tratamiento aplicado permitió una rápida degradación de los grupos cromóforos y hubo una gran influencia de la concentración de peróxido de hidrógeno en la velocidad de esta degradación. Los resultados fueron satisfactorios, ya que se eliminó la coloración y los productos de oxidación identificados no presentan toxicidad y pueden tratarse fácilmente por métodos tradicionales.

Palabras clave: Tintes; Antraquinona; Electro-Fenton; Efluente industrial; Proceso oxidativo avanzado.

\section{Introdução}

Lentes oftálmicas coloridas já estão no mercado há algum tempo e as tendências da moda impulsionam este produto cada vez mais, fazendo com que a variedade de cores disponíveis aumente a cada ano. Como consequência, as empresas oftálmicas precisam produzir tais produtos com mais frequência e em grande escala, chegando até mesmo a criar novos tons para lentes. A tonalidade das lentes solares é atingida através da imersão dessas em soluções contendo uma mistura de corantes, os quais penetram profundamente na superfície da lente resultando em tonalidades sólidas, graduadas, degradê ou com finalidades médicas. Geralmente são usados os chamados corantes dispersos, que são poucos solúveis em água e aplicados na forma de dispersões. As substâncias químicas coloridas contribuem significativamente com a poluição de recursos hídricos, pois dificultam a penetração dos raios solares, prejudicando, assim, o metabolismo fotossintético de algumas espécies, além de possuírem, em sua maioria, características recalcitrantes e cancerígenas (Peternel et al., 2006; Rêgo et al., 2014).

Este trabalho é uma parceria com uma empresa de produtos oftálmicos que produz resíduos contendo uma mistura de três corantes dispersos: azul 56, amarelo 54 e vermelho 60, os quais pertencem à classe das antraquinonas. A química específica do grupo da antraquinona é baseada no antraceno e consiste em três anéis de benzeno fundidos (estrutura básica do antraceno) com dois grupos carbonil no anel central. Esta estrutura é naturalmente incolor, mas a substituição dos anéis aromáticos resulta na coloração e controla sua intensidade (Gregory, 2003).

Os corantes do grupo das antraquinonas representam sérios problemas ambientais, já que não são biodegradáveis e apresentam toxicidade, o que torna necessária a aplicação de processos avançados para a descoloração e degradação desses compostos antes do descarte em corpos hídricos. É conhecido que todo método de tratamento apresenta vantagens e desvantagens em relação a eficiência sob diferentes condições, a praticidade, os requisitos de pré e pós-tratamento, o impacto ambiental e o custo. Dada esta complexidade, um único método raramente pode satisfazer essas demandas simultaneamente, sendo necessária uma combinação de métodos disponíveis e em desenvolvimento, maximizando assim seus pontos fortes e minimizando suas desvantagens (Kaushik \& Malik, 2009; Robinson et al., 2001; Routoula \& Patwardhan, 2020).

Dentre os processos químicos não tradicionais pode-se citar os processos eletroquímicos, que são capazes de oxidar ou reduzir íons metálicos, cianetos, compostos organoclorados, hidrocarbonetos aromáticos e alifáticos (Brillas, 2021). As vantagens desse processo dizem respeito a diminuição de produtos químicos para o tratamento, assim como a possibilidade de mineralização dos compostos orgânicos recalcitrantes junto a um alto poder de desinfecção (Maia et al., 2017). Vale ressaltar que o processo eletroquímico também pode ser combinado a vários outros para a maximização de sua eficiência, como os processos oxidativos avançados (POAs), chamados de "processos eletroquímicos oxidativos avançados (PEOAs)". Esses processos baseiam-se na produção de radicais hidroxilas (HO'), os quais são altamente oxidantes e não seletivos, sendo capazes de degradar compostos de difícil oxidação (Forti et al., 2020; Rêgo, et al., 2014; Tadayozzi et al., 2021). Uma das maneiras mais eficientes de gerar radicais hidroxilas é por meio de uma mistura de peróxido de hidrogênio e sais ferrosos, conhecida por "Reagente de Fenton" (Haber e Weiss, 1934), onde os radicais são produzidos em reações em cadeia (Feng et al., 2020; Guimarães, 2013; Zhang et al., 2019). Nesse contexto, o objetivo deste trabalho foi aplicar o processo oxidativo denominado 
eletro-Fenton para tratar um efluente industrial proveniente da fabricação de lentes solares, que contem diferentes corantes do grupo antraquinona em sua composição.

\section{Metodologia}

Quanto à abordagem, o trabalho foi desenvolvido sob uma perspectiva quantitativa (Martins, 2010), que se desenvolveu de acordo com as etapas descritas a seguir.

\section{Amostra de efluente}

O efluente é proveniente do setor de fabricação de lentes para óculos solares de uma empresa de produtos oftálmicos situada no interior do estado de São Paulo, Brasil. A amostra foi coletada e armazenada seguindo-se os preceitos do Guia de Coleta e Preservação de Amostras (CETESB, 2011). Dessa amostra de efluente foi preparada uma solução estoque de $2 \mathrm{~g} / \mathrm{L}$ e os estudos foram realizados fixando-se a concentração final do efluente em $100 \mathrm{mg} / \mathrm{L}$ na célula eletroquímica.

\section{Preparação dos eletrodos de óxidos}

A partir de resinas precursoras de rutênio, titânio e estanho (Forti, et al, 2001), foram construídos eletrodos de composição nominal Ti/ $\mathrm{Ru}_{0,3} \mathrm{Ti}_{0,5} \mathrm{Sn}_{0,2} \mathrm{O}_{2}$. A espessura teórica da camada ativa do óxido foi de $2 \mu \mathrm{m}$, para tal, foi realizado o controle da massa a ser depositada levando-se em consideração, o volume da camada, a fração molar de cada óxido e suas respectivas densidades.

Para o preparo dos eletrodos de óxidos, as placas de titânio de $2 \mathrm{~cm}^{2}$ foram tratadas previamente com o objetivo de aumentar a rugosidade, possibilitando maior aderência da camada de óxidos ao substrato de titânio. Estas placas de titânio foram jateadas com limalha de ferro; lavadas com bastante água; aquecidas durante 30 minutos em água deionizada; mergulhadas em isopropanol e deixadas em ultrassom por 30 minutos; lavadas com água deionizada; aquecidas durante 20 minutos em $\mathrm{HCl} 20 \%$; lavadas novamente com água; aquecidas durante 20 minutos em ácido oxálico 10\%; lavadas e secadas em corrente de ar quente. Após o tratamento das placas de titânio, estas foram, imediatamente, pesadas e pinceladas com as soluções precursoras, secas em corrente de ar quente e calcinadas a $400^{\circ} \mathrm{C}$, com fluxo constante de $5 \mathrm{dm}^{3} \mathrm{~min}^{-1} \mathrm{de}_{2}$, por 5 minutos. Após a obtenção da massa de óxido desejada, procedeu-se a calcinação final durante 1 hora a $400^{\circ} \mathrm{C}$ sob fluxo constante de $\mathrm{O}_{2}$. Os eletrodos obtidos foram fixados em um tubo de vidro.

$\mathrm{O}$ eletrodo de óxidos foi inserido em uma célula eletroquímica de compartimento único contendo $100 \mathrm{~mL}$ de $\mathrm{H}_{2} \mathrm{SO}_{4}$ 0,1 $\mathrm{mol} / \mathrm{L}$ e dois eletrodos auxiliares de platina (espiral) para condicionamento inicial, a fim de ativar os sítios ativos da superfície eletródica. Esse procedimento foi realizado por meio de voltametrias cíclicas (50 ciclos, no intervalo de 0 a $1,1 \mathrm{~V}$ vs. ECS com velocidade de varredura de $50 \mathrm{mV} / \mathrm{s}$ ), usando um bipotenciostato/galvanostato da Dropsens (DRP-STAT400).

\section{Processo eletro-Fenton}

O processo denominado eletro-Fenton conta com a junção da oxidação por meio de eletrodo (oxidação anódica) e do reagente de Fenton. Para aplicação desse processo inseriu-se o eletrodo $T i / \mathrm{Ru}_{0,3} \mathrm{Ti}_{0,5} \mathrm{Sn}_{0,2} \mathrm{O}_{2}$ e dois eletrodos auxiliares de platina em uma célula eletroquímica contendo como eletrólito de suporte $\mathrm{Na}_{2} \mathrm{SO}_{4} 0,05 \mathrm{~mol} / \mathrm{L}(\mathrm{pH}$ 3), solução do efluente 100 $\mathrm{mg} / \mathrm{L}$ e também o reagente de Fenton, variando-se as concentrações de $\mathrm{Fe}^{2+}\left(\mathrm{Fe}_{2} \mathrm{SO}_{4} .7 \mathrm{H}_{2} \mathrm{O}\right.$ ) e de $\mathrm{H}_{2} \mathrm{O}_{2} 29 \%$ (v/v), de acordo com a Tabela 1. O volume final da célula foi de $50 \mathrm{~mL}$. As eletrólises foram realizadas aplicando-se uma corrente de 50 $\mathrm{mA} / \mathrm{cm}^{2}$ durante 2 horas e foram realizadas em triplicata. 
Tabela 1. Concentrações dos reagentes de Fenton usados nos diferentes experimentos realizados.

\begin{tabular}{ccc}
\hline Experimentos & $\mathbf{F e}^{\mathbf{2 +}}(\mathbf{m g} / \mathbf{L})$ & $\mathbf{H}_{\mathbf{2}} \mathbf{O}_{\mathbf{2}}(\mathbf{m g} / \mathbf{L})$ \\
\hline $\mathbf{1}$ & 20 & 400 \\
$\mathbf{2}$ & 20 & 600 \\
$\mathbf{3}$ & 30 & 400 \\
$\mathbf{4}$ & 30 & 600 \\
\hline
\end{tabular}

Fonte: Autores.

\section{Análises de acompanhamento}

Durante as eletrólises foram coletadas amostras de $3 \mathrm{~mL}$ nos tempos $0,15,30,45,60,90$ e 120 minutos para leitura em espectrofotômetro UV-Visível em comprimento de onda de $545 \mathrm{~nm}$. Para essas leituras utilizou-se um Espectrofotômetro UV-1800, Shimadzu.

Também analisou-se usando a Cromatografia Líquida de Alta Eficiência (CLAE) utilizando um Shimadzu (Kyoto, Japan) Prominence LC 20 AT contendo um forno a CTO-10AS, um autosampler SIL 20A, um detector de comprimento de onda variável SPD-10A e um detector por índice de refração RID-20A. As separações foram efetuadas em uma coluna CLCODS (M) C-18 (250 x 4,6 mm i.d.; $5 \mu \mathrm{m}$ ), protegida por uma pré-coluna Supelcosil C-18 column (4 x 3,0 mm i.d.; $5 \mu \mathrm{m})$. A fase móvel utilizada foi uma mistura 70:20:10 (v/v) de acetonitrila, tampão acetato de amônio $(0,02 \mathrm{M})$ com 0,8\% de ácido tricloroacético ( $\mathrm{pH} 2,5)$ e metanol. A temperatura do forno foi de $25^{\circ} \mathrm{C}$; o fluxo de $1,2 \mathrm{~mL} \mathrm{~min}^{-1}$; volume de injeção de $30 \mathrm{~mL}$ e a detecção no UV em 215 e $254 \mathrm{~nm}$. Os cromatogramas foram registrados e integrados pelo software Lab-Solutions. A formação de ácidos de cadeia curta (sub-produtos) foi analisada no mesmo equipamento usando uma coluna Aminex HPX 87H (300 x 7,8 mm) a $35^{\circ} \mathrm{C}$. As amostras foram eluídas com $\mathrm{H}_{2} \mathrm{SO}_{4} 4 \mathrm{mM}$ a uma vazão de $0,6 \mathrm{~mL} \mathrm{~min}{ }^{-1}$ e detecção em $210 \mathrm{~nm}$.

A quantidade de carbono orgânico total inicial e final foram determinadas pelo equipamento TOC-VCPN Analyser Shimadzu.

\section{Resultados e Discussão}

A Figura 1 mostra o decaimento da concentração inicial do efluente na célula $(100 \mathrm{mg} / \mathrm{L})$ em função do tempo de aplicação do processo eletro-Fenton, para diferentes concentrações do reagente de Fenton, de acordo com a Tabela 1. 
Figura 1. Decaimento da concentração inicial do efluente em função do tempo de aplicação do processo eletro-Fenton, variando-se as concentrações dos reagentes de Fenton de acordo com a tabela 1. Eletrodo de composição nominal $\mathrm{Ti} / \mathrm{Ru}_{0,3} \mathrm{Ti}_{0,5} \mathrm{Sn}_{0,2} \mathrm{O}_{2}$.

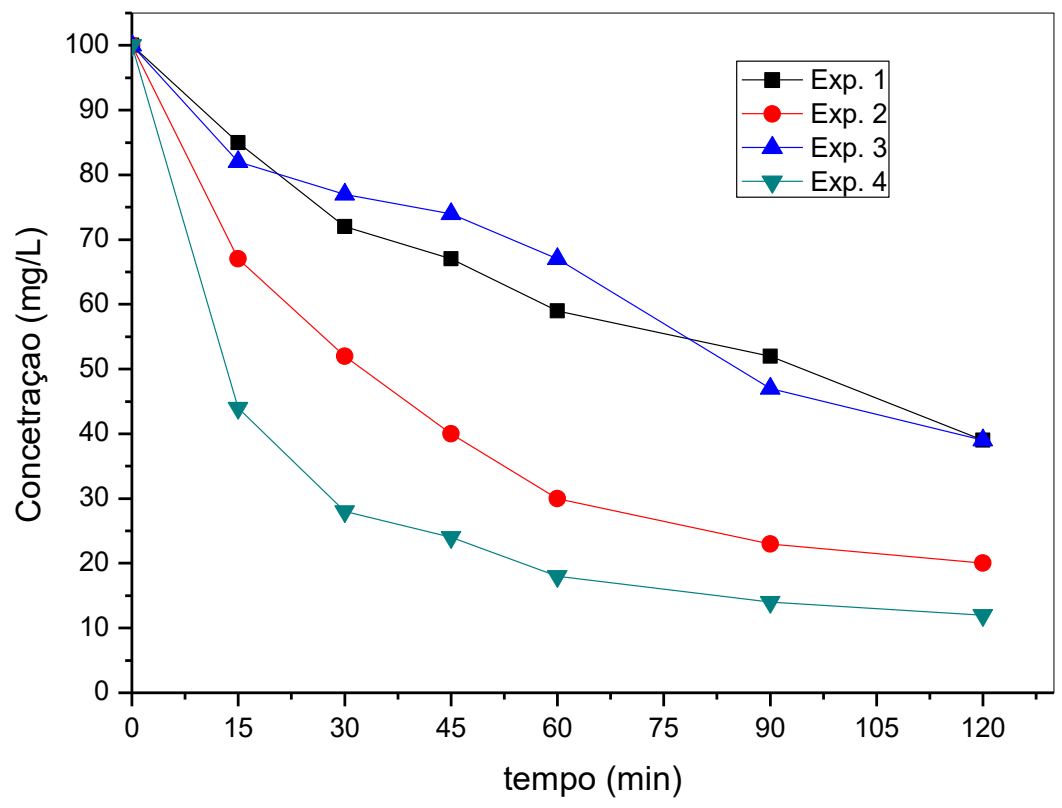

Fonte: Autores.

Esses resultados da Figura 1, obtidos por espectrometria UV-Vis, mostram que a concentração do reagente de Fenton influencia diretamente na eficiência do tratamento. Nota-se que nos experimentos com concentrações maiores de $\mathrm{H}_{2} \mathrm{O}_{2}(600$ mg/L, exp. 3 e 4) houve maior eficiência, ou seja, quanto mais $\mathrm{H}_{2} \mathrm{O}_{2}$ estiver disponível na solução, maior será a geração dos radicais hidroxilas (HO'), que são os responsáveis pela oxidação dos compostos orgânicos. No experimento 4, com concentrações maiores de ferro e de peróxido de hidrogênio (30:600) a oxidação dos corantes foi mais efetiva e rápida, alcançando 44\% de descoloração em 15 minutos de tratamento.

$\mathrm{O}$ uso eficiente de $\mathrm{H}_{2} \mathrm{O}_{2}$ implica na formação constante de $\mathrm{HO}^{\bullet}$ (produzido a partir da Eq. (1), do mecanismo simplificado de Fenton) e, consequentemente, na oxidação eficiente dos compostos orgânicos (como os corantes) em subprodutos (Eq. (2)) que também podem ser oxidados até compostos de cadeia simples (Eq. (3)). O excesso de peróxido de hidrogênio também pode ser consumido por reações ineficientes (reações nas quais HO• não é produzido), como mostra as equações (5) e (6). Como não foi obtido peróxido residual após os experimentos, pode-se concluir que o uso desse reagente foi eficiente. Além disso, também não houve a formação de lodo nas condições estudadas.

$$
\begin{array}{ll}
\mathrm{Fe}^{2+}+\mathrm{H}_{2} \mathrm{O}_{2} \rightarrow \mathrm{Fe}^{3+}+\mathrm{HO}^{\bullet}+\mathrm{HO}^{-} & \text {Eq. (1) } \\
\mathrm{HO}+\text { orgânicos } \rightarrow \text { subprodutos } & \text { Eq. (2) } \\
\text { Subprodutos }+\mathrm{HO}^{\bullet}+\rightarrow \text { inorgânicos } & \text { Eq. (3) } \\
\mathrm{Fe}^{3+}+\mathrm{H}_{2} \mathrm{O}_{2} \rightarrow \mathrm{Fe}^{2+}+\mathrm{HO}_{2} \cdot+\mathrm{H}^{+} & \text {Eq. (4) }
\end{array}
$$




$$
\begin{array}{ll}
2 \mathrm{H}_{2} \mathrm{O}_{2} \rightarrow 2 \mathrm{H}_{2} \mathrm{O}+\mathrm{O}_{2} & \text { Eq. (5) } \\
\mathrm{HO}^{\cdot}+\mathrm{H}_{2} \mathrm{O}_{2} \rightarrow \mathrm{H}_{2} \mathrm{O}+\mathrm{HO}_{2} & \text { Eq. (6) }
\end{array}
$$

A Figura 2 mostra imagens do efluente em tempo 0 e após 2 horas de aplicação do processo eletro-Fenton para os experimentos 2 e 4. Essas imagens mostram visualmente a descoloração do efluente causada pela aplicação dos processos eletro-Fenton, os quais causaram a oxidação dos grupos cromóforos presentes nas moléculas dos corantes.

Figura 2. Imagens do efluente em condições iniciais e após 2 horas de oxidação aplicando o processo eletro-Fenton usando 20 $\mathrm{mg} / \mathrm{L}$ de $\mathrm{Fe}^{2+}+600 \mathrm{mg} / \mathrm{L}$ de $\mathrm{H}_{2} \mathrm{O}_{2}$ (Exp. 2) e $30 \mathrm{mg} / \mathrm{L} \mathrm{de} \mathrm{Fe}^{2+}+600 \mathrm{mg} / \mathrm{L} \mathrm{de} \mathrm{H}_{2} \mathrm{O}_{2}$ (Exp. 4).
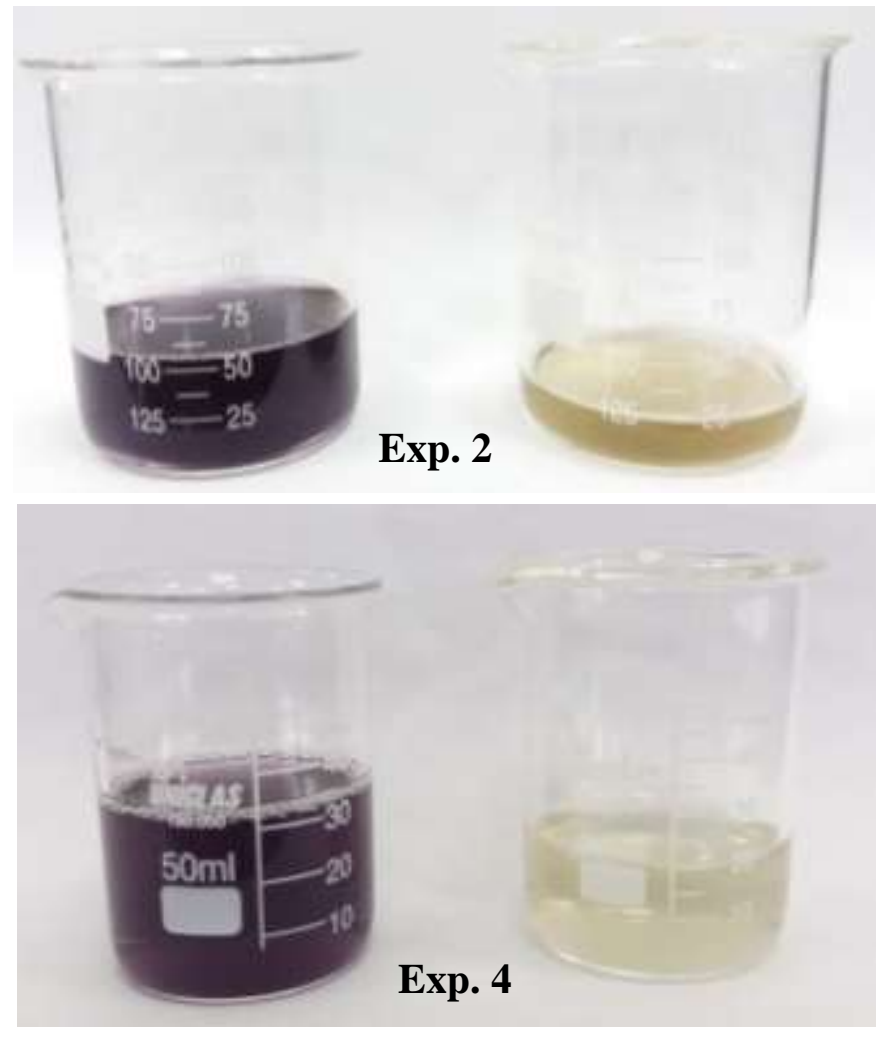

Fonte: Autores.

Essas imagens mostram visualmente a descoloração do efluente causada pela aplicação dos processos eletro-Fenton, os quais causaram a oxidação dos grupos cromóforos presentes nas moléculas dos corantes. Segundo Panizza \& Cerisola (2009), a análise UV-Vis indicou que a descoloração da solução de corantes sintéticos ocorre simultaneamente com a destruição dos anéis aromáticos produzindo intermediários incolores (principalmente ácido ftálico e pequenas espécies de carbonila) e depois o dióxido de carbono.

Por meio das análises por CLAE foi possível determinar os seguintes compostos presentes no efluente em condições iniciais: hidroxi-3-quinoftalona, 1,5-diaminobromo-4,8-dihidroxiantraquinona e 1-amino-4-hidroxi-2-fenoxi-antraquinona. Estas antraquinonas, referem-se aos corantes dispersos conhecidos como amarelo 54, azul 56 e vermelho 60, respectivamente. A concentração desses corantes foi monitorada por CLAE durante a aplicação do processo eletro-Fenton. Para todas as condições experimentais estudadas, obteve-se a degradação completa dos três corantes iniciais presentes no efluente industrial em até 60 minutos de aplicação do processo eletro-Fenton, como pode ser observado na Figura 3, que mostra o resultado 
representativo para o Exp. 2. Também pelo equipamento de CLAE foram identificados como principais subprodutos da oxidação das antraquinonas os ácidos fórmico, oxálico e acético.

Figura 3. Decaimento da concentração inicial dos corantes amarelo 54 (hidroxi-3-quinoftalona), azul 56 (1,5-diaminobromo4,8-dihidroxiantraquinona) e vermelho 60 (1-amino-4-hidroxi-2-fenoxi-antraquinona) presentes no efluente industrial em função do tempo de aplicação do processo eletro-Fenton, exp. 2.

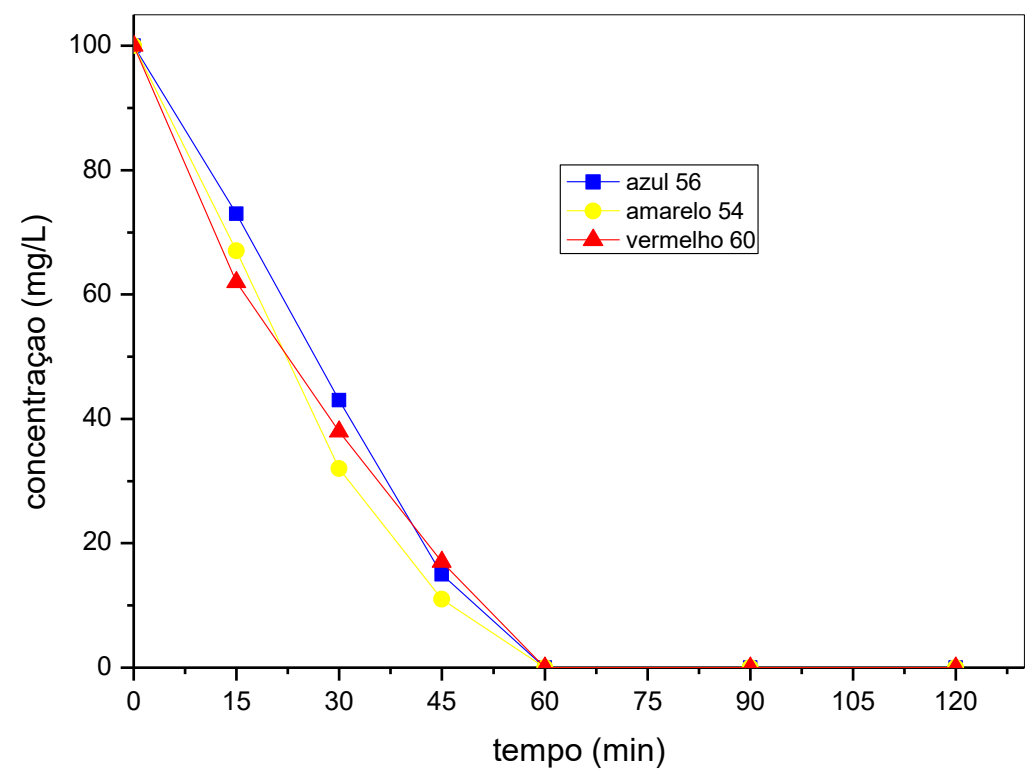

Fonte: Autores.

Os corantes da classe das antraquinonas possuem cinéticas de degradação distintas, atribuído à presença de grupos substituintes diferentes no anel aromático. De modo geral, a estrutura principal das antraquinonas geralmente é dividida em derivados aromáticos mais simples, permitindo uma manipulação mais fácil, ou seja, permitindo a aplicação de tratamentos químicos convencionais.

De acordo com Routoula e Patwardhan (2020), os tratamentos eletroquímicos das antraquinonas resultam em produtos geralmente com menor massa molecular e com maior potencial de mineralização comparado aos tratamentos biológicos. Os subprodutos mais comuns identificados foram fenol, ácido ftálico e ácidos carboxílicos de cadeia curta, formados, principalmente, pela clivagem e degradação subsequente do anel de antraquinona em várias etapas (Routoula \& Patwardhan, 2020).

Os resultados da análise de carbono orgânico total (COT) das amostras de efluente inicial e final (após 2h de aplicação dos tratamentos) estão mostrados na Tabela 2.

Tabela 2. Taxas de remoção (\%) de carbono orgânico total do efluente industrial após aplicação do processo eletro-Fenton.

\begin{tabular}{cc}
\hline Experimentos & COT (\% de remoção) \\
\hline $\mathbf{1}$ & $66,2 \pm 0,02$ \\
$\mathbf{2}$ & $72,5 \pm 0,01$ \\
$\mathbf{3}$ & $68,6 \pm 0,01$ \\
$\mathbf{4}$ & $76,4 \pm 0,02$ \\
\hline
\end{tabular}

Fonte: Autores. 
De acordo com o princípio dessa técnica de análise, ocorre a clivagem (decomposição) das moléculas orgânicas e a liberação do carbono em uma forma molecular simples $\left(\mathrm{CO}_{2}\right)$ que é ser quantificado. A análise de COT considera as parcelas biodegradáveis e não biodegradáveis da matéria orgânica, não sofrendo interferência de outros átomos que estejam ligados à estrutura orgânica, quantificando apenas o carbono presente na amostra. Sendo assim, observa-se pela Tabela 2 que todas as condições experimentais estudadas apresentaram taxas de remoção de carbono orgânico total acima de $50 \%$, ou seja, grande parte dos compostos orgânicos foram degradados após a aplicação do processo eletro-Fenton.

O processo eletro-Fenton constitui um dos mais poderosos processos oxidativos avançados com várias vantagens práticas, como eficiência de oxidação e poder de mineralização pela considerável formação dos radicais hidroxilas, além do fácil manuseio e possibilidade de tratar efluentes com grande faixa de concentração (Oturan, 2021). A combinação de processos resulta no efeito sinérgico e com isso o aumento na efetividade da oxidação dos compostos orgânicos (Brillas et al., 2009).

\section{Conclusão}

Nos estudos envolvendo a degradação de corantes presentes em efluente proveniente de uma indústria oftálmica, ficou evidenciada a elevada capacidade de degradação do processo eletro-Fenton. Aplicado em condições experimentais otimizadas ( 20 ou $30 \mathrm{mg} / \mathrm{L}$ de Fe$e^{2+}+600 \mathrm{mg} / \mathrm{L}$ de $\mathrm{H}_{2} \mathrm{O}_{2}$ ) - experimentos 2 e 4) obteve-se rápida e satisfatória degradação dos compostos orgânicos iniciais, bem como dos grupos cromóforos dos corantes. A concentração de $\mathrm{H}_{2} \mathrm{O}_{2}$ influencia diretamente na eficiência do reagente de Fenton devido a maior produção dos radicais hidroxilas (HO'), que são os responsáveis pela oxidação da matéria orgânica. Foi obtida a remoção da maior parte dos compostos orgânicos refratários presentes no efluente industrial, restando compostos de cadeia curta e baixa massa molecular, os quais podem ser tratados por tratamentos químicos tradicionais. O processo eletro-Fenton permite eficiência na degradação, rapidez e requer pequeno espaço físico para ser instalado. Apresenta custo médio, porém a relação custo/benefício de sua aplicação é expressiva.

Para trabalhos futuros, os autores pretendem aplicar outros processos oxidativos concomitantemente, com radiação UV e minimizar o uso de reagentes, a fim de melhorar a eficiência e a relação custo/benefício do processo de tratamento.

\section{Agradecimentos}

Os autores agradecem a PROPe/UNESP - Programa Institucional de Iniciação Científica para o Ensino Médio (PIBIC EM) pela bolsa concedida.

\section{Referências}

Brillas, E., (2021). Recent development of electrochemical advanced oxidation of herbicides. A review on its application to wastewater treatment and soil remediation. Journal of Cleaner Production, 290, 125841. https://doi.org/10.1016/j.jclepro.2021.125841

Brillas, E., Sirés, I., \& Oturan, M. A. (2009). Electro-Fenton process and related electrochemical technologies based on Fenton's reaction chemistry. Chemical reviews, 109(12), 6570-6631. https://doi.org/10.1021/cr900136g.

CETESB, Companhia Ambiental do Estado de São Paulo (2011). Guia nacional de coleta e preservação de amostras: água, sedimento, comunidades aquáticas e efluentes líquidos. 396 p. https://capacitacao.ana.gov.br/conhecerh/handle/ana/2211

Feng, D., Soric, A., \& Boutin, O. (2020). Treatment technologies and degradation pathways of glyphosate: A critical review. Science of The Total Environment, 140559. https://doi.org/10.1016/j.scitotenv.2020.140559

Forti, J. C., Loretti, G. H., Tadayozzi, Y. S., \& de Andrade, A. R. (2020). A phytotoxicity assessment of the efficiency 2, 4-D degradation by different oxidative processes. Journal of Environmental Management, 266, 110588. https://doi.org/10.1007/s12678-010-0020-3

Forti, J. C., Olivi, P., \& de Andrade, A. R. (2001). Characterisation of $\mathrm{DSA}^{\circledR}$-type coatings with nominal composition $\mathrm{Ti}^{-} / \mathrm{Ru}_{0.3} \mathrm{Ti}_{(0.7-\mathrm{x})} \mathrm{Sn}_{\mathrm{x}} \mathrm{O}_{2} \mathrm{prepared}_{\mathrm{via}} \mathrm{a}$ polymeric precursor. Electrochimica Acta, 47(6), 913-920. https://doi.org/10.1016/S0013-4686(01)00791-5 
Research, Society and Development, v. 10, n. 11, e108101119397, 2021

(CC BY 4.0) | ISSN 2525-3409 | DOI: http://dx.doi.org/10.33448/rsd-v10i11.19397

Gregory, P. (2003). Important chemical chromophores of dye classes. Industrial Dyes: Chemistry, Properties, Applications, 35-39. https://doi.org/10.1002/3527602011.ch2

Guimarães, J.R. (2013). Processos Oxidativos Avançados. Revista TAE, 32-36. https://www.revistatae.com.br/Artigo/355/processos-oxidativos-avancados

Haber, F., \& Weiss, J. (1934). The catalytic decomposition of hydrogen peroxide by iron salts. Proceedings of the Royal Society of London. Series AMathematical and Physical Sciences, 147(861), 332-351. https://doi.org/10.1098/rspa.1934.0221

Kaushik, P., \& Malik, A. (2009). Fungal dye decolourization: recent advances and future potential. Environment International,35(1), 127-141. https://doi.org/10.1016/j.envint.2008.05.010

Maia, L. C. C., Jambo, H. C. M., \& Gomes, J. A. D. C. P. (2018). Tratamento eletroquímico de efluentes industriais-alternativa para a remoção de contaminantes e potencial aproveitamento de H 2. Matéria (Rio de Janeiro), 22. https://doi.org/10.1590/S1517-707620170005.0259

Martins, R. A. (2010). Abordagens quantitativa e qualitativa. Metodologia de pesquisa em engenharia de produção e gestão de operações. Rio de Janeiro: Elsevier, 47-62. http://eu-ireland-custom-media-prod.s3.amazonaws.com/Brasil/Downloads/14-10/mztodologia.pdf

Oturan, M. A. (2021). Outstanding performances of the BDD film anode in electro-Fenton process: Applications and comparative performance. Current Opinion in Solid State and Materials Science, 25(3), 100925. https://doi.org/10.1016/j.cossms.2021.100925

Panizza, M., \& Cerisola, G. (2009). Electro-Fenton degradation of synthetic dyes. Water research,43(2), 339-344. https://doi.org/10.1016/j.watres.2008.10.028

Peternel, I., Koprivanac, N., \& Kusic, H. (2006). UV-based processes for reactive azo dye mineralization. Water Research,40(3), 525-532. https://doi.org/10.1016/j.watres.2005.11.029

Rêgo, F. E. F., Solano, A. M. S., da Costa Soares, I. C., da Silva, D. R., Huitle, C. A. M., \& Panizza, M. (2014). Application of electro-Fenton process as alternative for degradation of Novacron Blue dye. Journal of Environmental Chemical Engineering, 2(2), 875-880. https://doi.org/10.1016/j.jece.2014.02.017

Robinson, T., McMullan, G., Marchant, R., \& Nigam, P. (2001). Remediation of dyes in textile effluent: a critical review on current treatment technologies with a proposed alternative. Bioresource Technology, 77(3), 247-255. https://doi.org/10.1016/S0960-8524(00)00080-8

Routoula, E., \& Patwardhan, S. V. (2020). Degradation of anthraquinone dyes from effluents: a review focusing on enzymatic dye degradation with industrial potential. Environmental Science \& Technology, 54(2), 647-664. https://doi.org/10.1021/acs.est.9b03737

Tadayozzi, Y. S., Santos, F. A. D., Vicente, E. F., \& Forti, J. C. (2021). Application of oxidative process to degrade paraquat present in the commercial herbicide. Journal of Environmental Science and Health, Part B, 1-5. https://doi.org/10.1080/03601234.2021.1936991

Zhang, M. H., Dong, H., Zhao, L., Wang, D. X., \& Meng, D. (2019). A review on Fenton process for organic wastewater treatment based on optimization perspective. Science of the total environment, 670, 110-121. https://doi.org/10.1016/j.scitotenv.2019.03.180 